\section{\$21. Small-scale Dynamo Action in Multi-scale Magnetohydrodynamic and Micro-turbulence}

\section{Li, J.Q., Kishimoto, Y. (Kyoto Univ.)}

The coexistence of different scale electrostatic(ES) and electromagnetic (EM) fluctuations is common in plasmas. One important issue is on the nonlinear interaction mechanism and the exchange channel of kinetic and magnetic energy in mixed MHD and micro-scale turbulence. Of particular interest is the magnetic island dynamics in plasma turbulence. On one hand, the effects of magnetic fluctuations including islands on the turbulent transport have been intensively investigated based on theory/simulation to understand the confinement improvement. On the other hand, the diamagnetic drift coupled with the magnetic fluctuation may alter the island evolution. Here, a smallscale dynamo action induced by the micro-turbulence is reported based on direct Landau-fluid simulation of mixed resistive MHD and ITG turbulence. A twisting oscillation of magnetic island (hereafter referred to as magnetic island seesaw) pivoting around the singular surface is visibly observed. Furthermore, a minimal model proposed can elucidate the underlying mechanism.

Simulations are performed using an initial value code in a current sheet [1]. A prominent magnetic island oscillation is commonly observed in the final nonlinear stage with a fully reconnected island, showing a dynamic quasi-steady state. A typical simulation shows an island oscillation, which is visualized by a movie of island evolution, and much more clearly, by snapshots of the dominant flux component $m=1$. The oscillation occurs as pivoting along the singular surface like a seesaw (referred to hereafter as an island seesaw). To explore the energy exchange channel between micro-turbulence and MHD behind the seesaw mechanism, the averaged EM torque

$$
<T_{E M y}>\hat{z}=\iint_{x y} x \hat{x} \times(\vec{j} \times \vec{B})_{y} d x d y / L_{x} L_{y},
$$

exerting on the island due to the fluctuating EM force in the $y$ direction is calculated. Simulations with a parametric scan[2] show that when $\eta_{i}$ is larger than a threshold $\eta_{i c}$, $\left.<T_{E M y}\right\rangle \hat{z}$ starts to exponentially increase and tends to oscillate in time as shown in Fig.1(a). The amplification of EM torque in the presence of strong micro-ITG results mainly from dramatic growth of fluctuating currents (and also magnetic fields) at small-scales as shown in Fig.1(b), which corresponds to the spatial scale of ITG turbulence. Note that the fluctuating currents at large-scales $\left(k_{y} \leq 0.3\right)$ have a little change, showing no more kinetic energy conversion into the magnetic energy at such scales. For comparison, Fig. 1(c) shows almost no increase of fluctuating currents at small-scales for small $\eta_{i}$ under the threshold $\eta_{i c}$. To reveal the driving mechanism of the small-scale fluctuating currents, Fig.1(d) plots time evolution of the intensity of magnetic induction for a representative component of $k_{y}=0.7$ in Fig.1(b), showing that the magnetic induction exponentially grows and finally saturates at almost the same level when $\eta_{i} \geq \eta_{i c}$. These features of exponential growth of fluctuating current and magnetic field at small-scales notably signify a small-scale dynamo action. Fig.1 logically exhibits the evidence of a small-scale dynamo action under the nonlinear interaction of micro-turbulence. Small-scale turbulent dynamos convert kinetic energy into magnetic energy so that the MHD dynamics are influenced. The island seesaw is just the resultant manifestation of the small-scale dynamo action in the multi-scale EM turbulence.
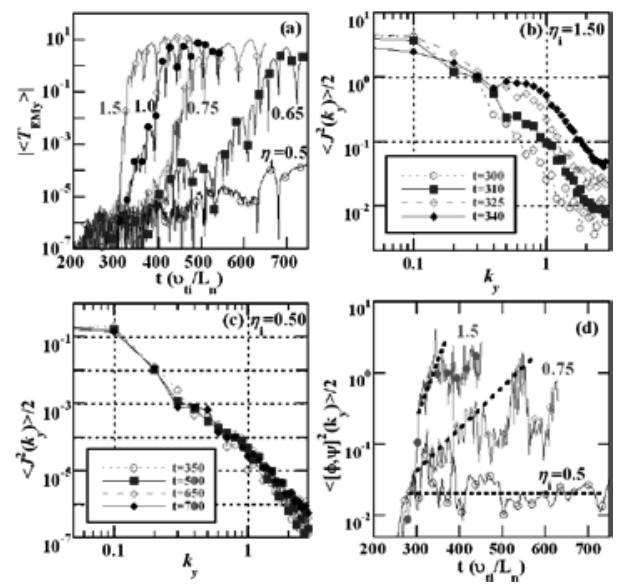

Fig.1 Evolution of the small-scale dynamo action versus ITG turbulence intensity. (a)-(d) corresponds to the averaged EM torque amplitude $\left|T_{E M y}\right|$; dynamo driving intensity $<[\phi, \psi]^{2}\left(k_{y}\right)>/ 2$ for a representative of $k_{y}=0.7$; and the $k_{y}$ spectra of fluctuating current for different ITG driving force.

To further clarify the generation of the small-scale dynamos in the direct simulation above, we propose a minimal model, which preserves most elementary MHD island dynamics and the multi-scale interaction with ES ITG mode. The model consists of the reduced MHD and an independently evolving ES ITG eigenmode representing the micro-turbulence. The modeling simulation well reproduces the observations in the full simulations. The parity analyses based on the minimal model show a dynamo action, which is characterized not only by a dynamo threshold as shown in Fig. 1 by $\eta_{i c}$, but also by a positive feedback of the flows to enhance the dynamo generation.

In summary, it is identified that the island seesaw is driven by a net oscillatory electromagnetic torque, which results from small-scale dynamo-generated current and magnetic field. The dynamo mechanism may offer an important energy exchange channel between MHD and micro-turbulence in magnetic fusion plasmas.

1) Li, J. Q. and Kishimoto, Y. et al.: Nucl. Fusion 49 (2009) 095007.

2) Li, J. Q. and Kishimoto, Y. Phys. Plasmas 19 (2012) 030705 . 